\title{
Vessel packaging effect in laser speckle contrast imaging and laser Doppler imaging
}

\author{
Ingemar Fredriksson \\ Marcus Larsson
}




\title{
Vessel packaging effect in laser speckle contrast imaging and laser Doppler imaging
}

\author{
Ingemar Fredriksson ${ }^{\mathrm{a}, \mathrm{b}, *}$ and Marcus Larsson ${ }^{\mathrm{a}}$ \\ aLinköping University, Department of Biomedical Engineering, Linköping, Sweden \\ berimed AB, Järfälla-Stockholm, Sweden
}

\begin{abstract}
Laser speckle-based techniques are frequently used to assess microcirculatory blood flow. Perfusion estimates are calculated either by analyzing the speckle fluctuations over time as in laser Doppler flowmetry (LDF), or by analyzing the speckle contrast as in laser speckle contrast imaging (LSCl). The perfusion estimates depend on the amount of blood and its speed distribution. However, the perfusion estimates are commonly given in arbitrary units as they are nonlinear and depend on the magnitude and the spatial distribution of the optical properties in the tissue under investigation. We describe how the spatial confinement of blood to vessels, called the vessel packaging effect, can be modeled in LDF and LSCI, which affect the Doppler power spectra and speckle contrast, and the underlying bio-optical mechanisms for these effects. As an example, the perfusion estimate is reduced by $25 \%$ for LDF and often more than $50 \%$ for LSCl when blood is located in vessels with an average diameter of $40 \mu \mathrm{m}$, instead of being homogeneously distributed within the tissue. This significant effect can be compensated for only with knowledge of the average diameter of the vessels in the tissue. $\odot$ The Authors. Published by SPIE under a Creative Commons Attribution 3.0 Unported License. Distribution or reproduction of this work in whole or in part requires full attribution of the original publication, including its DOI. [DOI: 10.1117/1.JBO.22.10.106005]
\end{abstract}

Keywords: microcirculation; blood flow; Monte Carlo simulations; modeling.

Paper 170421R received Jun. 30, 2017; accepted for publication Sep. 12, 2017; published online Oct. 10, 2017.

\section{Introduction}

Tissue vitality is strongly dependent on a functional microcirculation. Deficiencies in this circulation can result in ischemic conditions with an increased risk for tissue necrosis. With optical techniques, such as laser Doppler flowmetry (LDF) ${ }^{1}$ and laser speckle contrast imaging (LSCI) ${ }^{2}$ it is possible to objectively assess both spatial and temporal variations in the microcirculatory perfusion. Other optical techniques, where differences in the absorption characteristics of oxygenized and deoxygenized hemoglobin are utilized, enable the quantification of blood oxygen saturation. ${ }^{3,4}$

Common for most of these techniques is the challenge to make quantitative estimations of the microcirculatory parameters in the presence of other confounding components in the tissue. This includes not only the tissue composition of scattering and absorbing compounds but also the spatial distribution of tissue optical properties. Simulating photon propagation in layered tissue using the Monte Carlo technique clearly shows that a nonhomogeneous layer distribution of the optical properties affects the amount of backscattered photons. ${ }^{5-7}$ Similarly, when confining blood to vessels rather than assuming a homogeneous distribution, a significant effect on photon propagation and the amount of backscattered light can be noted. ${ }^{7-10}$

A common way to account for intra- and interindividual variations in optical and geometrical properties is to make use of algorithms based on inverse modeling. ${ }^{11-13}$ In order to make these algorithms effective and informative, the simulation of photon propagation needs to be accurate. In addition, the biooptical model needs to be representative for real tissue and

*Address all correspondence to: Ingemar Fredriksson, E-mail: ingemar. fredriksson@liu.se detailed enough to account for all effects that significantly affect the backscattered photons. Leaving out model parameters with a significant impact on photon transport will not only result in an increased model misfit but also in a decreased accuracy in other estimated parameters. ${ }^{5,6}$ Hence, the vessel packaging of absorbing hemoglobin needs to be taken into account when analyzing, e.g., the diffusely backscattered optical spectrum from skin using white-light spectroscopy. ${ }^{7,12}$ Similarly, the vessel packaging of moving red blood cells affects how photons are Doppler shifted. Hence, to properly consider this effect when analyzing optical signals originating from Doppler shifts (in, e.g., LDF and LSCI), an accurate model for vessel packaging of moving scatterers is needed.

We have previously shown that it is possible to accurately model laser Doppler power spectra in LDF using a multilayered tissue model. ${ }^{12,14-16}$ The vessel packaging effect in this model is further described and evaluated in this article. We have also shown how the speckle contrast can be calculated from the Doppler power spectrum, ${ }^{17}$ a fact that facilitates the investigation of the vessel packaging effect for LSCI.

During recent years, there has been an increased interest in studying how LSCI depends on various parameters, including optical and geometrical properties as well as the flow pattern. ${ }^{17-20}$ This paper is a continuation of that work that will ultimately lead to a better understanding and refined methods to translate the measured contrast into accurate perfusion measures.

The aim of this paper is to present a model for the vessel packaging effect in LDF and LSCI. The model is intended to be used when postprocessing Monte Carlo simulated data from tissue models where the blood is homogeneously distributed, rather than using models, where each and every vessel diameter is individually assessed. We describe the mechanisms 
that account for the effect and how it affects the conventional perfusion estimates generated by the techniques.

\section{Theory and Methods}

\subsection{Definition of Average Vessel Diameter}

Throughout this paper, the volume-weighted average vessel diameter is considered, rather than the number-weighted average. Hence, if we have four vessels with a diameter of $10 \mu \mathrm{m}$ and one with the diameter of $20 \mu \mathrm{m}$ within a certain volume, all with the same length $l$, the average vessel diameter of those five vessels is $15 \mu \mathrm{m}$ rather than $12 \mu \mathrm{m}$. Although not explicitly defined, the same interpretation of the average vessel diameter is done in previous papers on the topic, for example, Svaasand et al., ${ }^{8}$ van Veen et al., ${ }^{9}$ and Fredriksson et al. ${ }^{10}$

\subsection{Modeling Doppler Spectra}

Laser Doppler power spectra were calculated from tissue models, as previously described in Fredriksson et al., ${ }^{12}$ outlined in the flowchart in Fig. 1. The calculations were based on threelayered models of skin tissue with one epidermis layer of varying thickness $\left(t_{\text {epi }}\right)$, one upper dermis layer with a fixed thickness of $0.2 \mathrm{~mm}$, and one lower dermis with an infinite thickness. The reduced scattering coefficient $\left(\mu_{\mathrm{s}}^{\prime}\right)$ was the same for all three layers. The epidermis layer contained a varying volume fraction of melanin $\left(f_{\text {mel }}\right)$ as absorber and no blood, whereas the dermis layers contained blood of varying volume fraction $\left(f_{\text {blood, } 1}\right.$ and $f_{\text {blood,2 }}$, hemoglobin oxygen saturation $\left(S_{\mathrm{O}_{2}}\right)$, flow speed distribution $\left(P_{v}\right)$, and average vessel diameter $(D)$. The light absorption in the epidermis layer $\left(\mu_{\mathrm{a}, 0}\right)$ was given by the melanin, whereas the absorption in the dermis layers $\left(\mu_{\mathrm{a}, 1}\right.$ and $\left.\mu_{\mathrm{a}, 2}\right)$ was given by the volume fraction of blood and its oxygen saturation at the given wavelength.

The three-layered model was Monte Carlo simulated for various epidermis thicknesses and reduced scattering coefficients, where the pathlengths for all detected photons were stored and analyzed as pathlength distributions for each layer. For the three-layered model, six pathlength distributions were created, one describing the pathlength for light that had only been propagated in the first layer, one distribution over pathlengths in the first layer for light that had been propagating in the two first layers, and so on. The Monte Carlo simulations in Ref. 12 were based on a setup with optical fibers, but the same principle was used in this study for a camera setup with even illumination.

The effect of absorption, blood flow speed distribution, and average vessel diameter was added in a fast postprocessing step. In short, the intensity from each pathlength was reduced by applying Beer-Lambert's law with the given absorption in each layer. Single shifted optical Doppler spectra for each speed $v$ in the speed distribution were calculated, as described in Fredriksson and Larsson. ${ }^{17}$ These spectra were then successively cross-correlated taking into account different degrees of multiple Doppler shifts; i.e., the optical Doppler spectrum for light Doppler shifted exactly two times was calculated as the autocorrelation of the single-shifted spectrum, the spectrum for light shifted exactly three times was calculated as the two-times shifted spectrum cross-correlated with the single-shifted spectrum, and so on. The optical Doppler spectrum for non-Doppler shifted light was given by the Dirac delta function (i.e., all energy located at frequency 0 ).

The resulting multiple Doppler shifted spectra were calculated using the shift distributions, i.e., the distributions over the number of Doppler shifts the light undergoes for each pathlength in the pathlength distribution. First, a distribution over

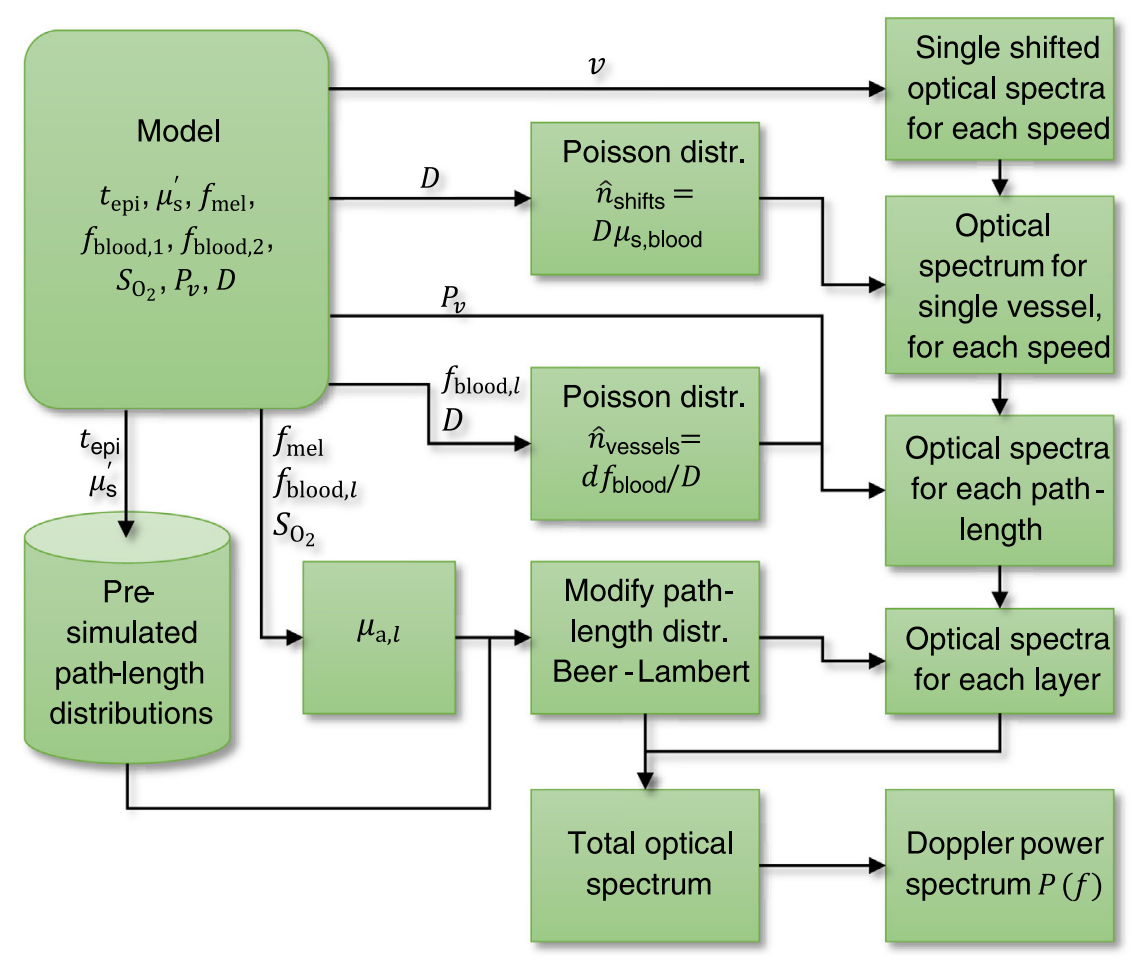

Fig. 1 Flowchart for calculating a Doppler power spectrum from the three-layered model with a given set of parameters. 
the number of Doppler shifts the light undergoes when crossing a single vessel is calculated. That distribution can be approximated by a Poisson distribution with the expectation value approximated by the average vessel diameter $D$ times the scattering coefficient of blood $\mu_{\mathrm{s} \text {,blood }}=222 \mathrm{~mm}^{-1}$ :

$$
\hat{n}_{\text {shifts }}=D \mu_{\text {s,blood }} \text {. }
$$

That distribution is used for calculating an optical Doppler spectrum for each speed in the speed distribution representing the spectra for passing through a single vessel. In reality, some light will cross the vessel in the periphery of the vessel, i.e., with a photon path shorter than the diameter, whereas some light will cross the vessel with a rather flat angle, i.e., with a path longer than the diameter. However, simulations have shown that a Poisson distribution with the expectation value given in Eq. (1) is a good approximation.

A second distribution was then calculated describing the number of vessels the light passes. For a given pathlength $d$, the number of vessels that the light passes through can be described by a Poisson distribution with the expectation value:

$\hat{n}_{\mathrm{vessels}}=\frac{d f_{\text {blood }}}{D}$

where $f_{\text {blood }}$ is the volume fraction of blood in the layer. This is intuitive, since the number of vessels scales with $f_{\text {blood }} / D^{2}$, whereas the probability to cross a vessel for a constant number of vessels scales with $d D$.

For light that had been propagating in both blood layers, the resulting optical Doppler spectrum was calculated as the crosscorrelation of the spectra from the first and second blood layers. The final optical Doppler spectrum was then calculated by weighting the optical Doppler spectra from the light that had been propagated in only the first layer, the first and second layer, and all three layers, depending on the intensity. The Doppler power spectrum $P(f)$ was finally calculated as the autocorrelation of the optical Doppler spectrum. A detailed

Table 1 Average, standard deviation, and median values of the model parameters in the 2000 random models. Row four is the average blood fraction in the two layers, whereas row five denotes the relationship between the blood fraction in the two layers. The two last rows give the average speed of the speed distribution $P_{V}$ and the width of the speed distribution in relation to the average speed, respectively.

\begin{tabular}{lccc} 
& Average & Standard deviation & Median \\
\hline$t_{\text {epi }}(\mu \mathrm{m})$ & 101 & 62 & 89 \\
$\mu_{\mathrm{s}}^{\prime}\left(\mathrm{mm}^{-1}\right)$ & 2.0 & 0.6 & 1.9 \\
$f_{\text {mel }}(\%)$ & 2.3 & 2.5 & 1.5 \\
$\left(f_{\text {blood }, 1}+f_{\text {blood }, 2}\right) / 2(\%)$ & 1.0 & 0.7 & 0.9 \\
$f_{\text {blood }, 1} / f_{\text {blood }, 2}(-)$ & 1.0 & 0.5 & 1.0 \\
$S_{\mathrm{O}_{2}}(\%)$ & 50 & 29 & 50 \\
$v(\mathrm{~mm} / \mathrm{s})$ & 1.2 & 0.8 & 1.0 \\
$\operatorname{std}(v) /\langle v\rangle(-)$ & 1.9 & 1.0 & 1.6 \\
\hline
\end{tabular}

mathematical description of the calculations of the Doppler power spectrum is found in Fredriksson et al. ${ }^{12}$

At first, a standard setup of the three-layered model was used. This standard model had an epidermal thickness of $75 \mu \mathrm{m}$, a reduced scattering coefficient of $3.0 \mathrm{~mm}^{-1}$ (anisotropy factor $g=0.8$ ), and absorption coefficients of $0.15,0.0043$, and $0.0043 \mathrm{~mm}^{-1}$ for the epidermis layer and the two blood layers, respectively. The distribution of flow speeds had an average speed of $1 \mathrm{~mm} / \mathrm{s}$ and was decreasing with speed. The vessel diameter parameter $D$ was varied between 5 and $100 \mu \mathrm{m}$.

In order to evaluate the vessel packaging effect in the more general case, 2000 three-layered models with random parameters $t_{\text {epi }}, \mu_{\mathrm{s}}^{\prime}, f_{\text {mel }}, f_{\text {blood }, 1}, f_{\text {blood }, 2}, S_{\mathrm{O}_{2}}$, and $P_{v}$ were generated. The distribution of the parameters in these 2000 models is summarized in Table 1.

\subsection{Validation Simulations of Discrete Vessel Models}

Two validation models were created and Monte Carlo simulated. Both models consisted of a homogeneous semi-infinite scattering layer with a refractive index of 1.4 and a reduced scattering coefficient $\mu_{\mathrm{s}}^{\prime}$ of $2.0 \mathrm{~mm}^{-1}$ containing various amounts of vessels (cylinder shaped) oriented parallel to the surface with random directions in that plane. The vessels all had a length of $20 \mathrm{~mm}$ and were randomly but homogeneously (on a large scale) distributed within a radius of $6 \mathrm{~mm}$ from the central axis of the model, at a random depth between 0 and $5 \mathrm{~mm}$. The light source had a diameter of $1.0 \mathrm{~mm}$ and illumined the model perpendicularly. All photons that were backscattered from the model within a radius of $3 \mathrm{~mm}$ and an angle of $0.3 \mathrm{rad}$ were detected. The medium outside the modeled tissue had a refractive index of 1.0. Hence, a part of the light was refracted in the surface and not detected. The blood in the vessels had a scattering coefficient $\mu_{\mathrm{s}}$ of $222 \mathrm{~mm}^{-1}$ and a Gegenbauer kernel scattering phase function ${ }^{21}$ with parameters $\alpha_{\mathrm{Gk}}=1.0$ and $g_{\mathrm{Gk}}=0.984$, resulting in an anisotropy factor $g$ of 0.991 and a reduced scattering coefficient $\mu_{\mathrm{s}}^{\prime}$ of $2.0 \mathrm{~mm}^{-1}$. The absorption coefficient $\mu_{\mathrm{a}}$ of the blood was set to $0.5 \mathrm{~mm}^{-1}$. These optical properties are valid at $780 \mathrm{~nm}$ for $50 \%$ oxygenated blood with a hematocrit of $43 \%$ and a mean cell hemoglobin concentration of $345 \mathrm{~g} / 1$ RBC. . $^{12,15}$

The first model contained 200 vessels all with a diameter of $50 \mu \mathrm{m}$. All vessels had a parabolic flow profile with average velocity of $1 \mathrm{~mm} / \mathrm{s}(2 \mathrm{~mm} / \mathrm{s}$ in the axial center of the vessel, 0 at the periphery). The blood vessels occupied $0.61 \%$ of the sampling volume in the model.

The second model contained 2000 vessels with vessel diameters having a volume weighted gamma distribution. The average velocity in the vessels was proportional to the diameter squared so that, for example, a vessel with a diameter of $50 \mu \mathrm{m}$ had an average velocity of $2 \mathrm{~mm} / \mathrm{s}$ and a vessel with a diameter of $10 \mu \mathrm{m}$ had an average velocity of $0.08 \mathrm{~mm} / \mathrm{s}$. The speed profile within each vessel was parabolic. The average vessel diameter in the model was $33 \mu \mathrm{m}$ with a standard deviation of $25 \mu \mathrm{m}$. The blood vessels occupied $1.0 \%$ of the sampling volume in the model and the average flow speed was $1.0 \mathrm{~mm} / \mathrm{s}$. A crosssectional view of this model is shown in Fig. 2.

\subsection{Laser Speckle Contrast Calculation}

In LSCI, the speckle contrast is used to estimate blood perfusion. Fredriksson and Larsson ${ }^{17}$ have previously described 


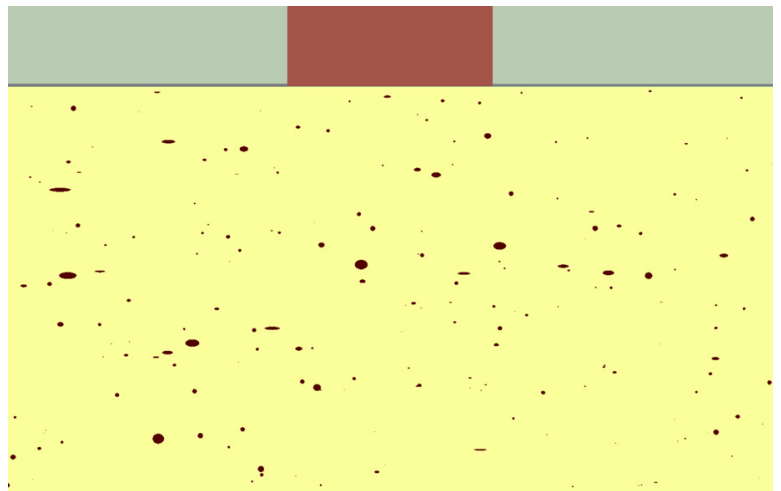

Fig. 2 Cross-sectional view of the simulated model with a gamma distribution of vessel diameters. The 1-mm diameter light source is shown in the top of the image. The dark ellipsoids represent crosssections of vessels, where the elongation of the ellipsoid depends on the angle relative to the cross-sectional plane.

how the speckle contrast can be calculated from a simulated Doppler power spectrum $P(f)$. In short, the intensity correlation function is calculated as

$g^{(2)}(\tau)=\left|\mathcal{F}^{-1}\{P(f)\}\right|$,

where $\mathcal{F}^{-1}$ is the inverse Fourier transform, $f$ is frequency, and $\tau$ is time. Using an extension of the Siegert relationship, ${ }^{22,23}$ valid for nonergodic medium, such as tissue, where only a fraction of the light has been Doppler shifted, gives the field correlation function:

$g^{(1)}(\tau)=\sqrt{1+g^{(2)}(\tau)-g^{(2)}(0)}$.

From this relationship, the speckle contrast $K$ as a function of exposure time $T$ is given by ${ }^{24,25}$

$K^{2}(T)=\frac{2 \beta}{T} \int_{0}^{T}\left|g^{(1)}(\tau)\right|^{2}\left(1-\frac{\tau}{T}\right) \mathrm{d} \tau$,

where $\beta$ is the coherence factor set to unity in this study.

\subsection{Perfusion Estimates}

In commercial LDF systems, the perfusion value is calculated from the first moment of the Doppler power spectrum, i.e.,

$$
\int_{0}^{f_{\max }} f P(f) \mathrm{d} f .
$$

In commercial LSCI systems, the perfusion value is calculated from the contrast $K$ at one single exposure time, for example, ${ }^{2}$

$$
\frac{1}{K^{n}}-1 \text {, }
$$

where $n$ usually equals 1 or 2 . In this study, the perfusion estimate calculated using Eq. (7) is calculated for contrasts for an exposure time of $6 \mathrm{~ms}$.

\section{Results}

\subsection{Vessel Packaging Model Validation}

Comparisons between optical Doppler spectra generated from the discrete vessel validation simulations (Sec. 2.3) and those calculated from the model described in Sec. 2.2, are found in Fig. 3, where "simulated" refers to the validation simulation and "modeled $\mathrm{xx} \mu \mathrm{m}$ " refers to calculated spectra from the model in Sec. 2.2. Corresponding comparisons of contrast as a function of exposure time are also shown in the same figure. In the first example, the validation simulation model contained 200 vessels, all with a diameter of $50 \mu \mathrm{m}$. The second validation simulation contained 2000 blood vessels with a gamma distributed diameter and diameter dependent flow speed, with an average diameter of $33 \mu \mathrm{m}$. To demonstrate the accuracy of the proposed vessel packaging model, spectra were calculated using a homogeneous model ( $0 \mu \mathrm{m}$ epidermis, same tissue fraction of blood in both dermis layer) with the same optical properties, speed distribution, and volume fraction of blood as that used in the validation simulations. To further demonstrate the vessel packaging effect, spectra were calculated from the homogeneous model using both the average diameter from the validation simulations and an infinitely small vessel diameter $(0 \mu \mathrm{m})$ to mimic homogeneously distributed blood. Frequencies up to $3 \mathrm{kHz}$ are shown because clear differences between spectra calculated with and without inclusion of the vessel packaging effect are foremost found at low frequencies. However, all Doppler power spectra in this study contained frequencies up to $25 \mathrm{kHz}$. The frequency resolution in the spectra is $49 \mathrm{~Hz}$ and the time resolution in the contrast curves is $0.02 \mathrm{~ms}$. The fraction of non-Doppler shifted light was $59 \%$ in the validation simulation in the first example (50 $\mu \mathrm{m}$ diameter), which should be compared to $56 \%$ in the homogeneous model including the vessel packaging effect, and $23 \%$ in the model with the homogeneously distributed blood. Corresponding numbers from the simulation containing a gamma distribution of vessel diameters in the simulation were $32 \%, 35 \%$, and $13 \%$, respectively.

\subsection{Effect on Perfusion Estimates}

The vessel packaging effect was studied in detail for vessel diameters ranging from 5 to $100 \mu \mathrm{m}$ in the standard model described in the second last paragraph of Sec. 2.2. As shown in the aforementioned examples, the most obvious change in the Doppler spectra when changing the vessel diameter is the fraction of non-Doppler shifted light, i.e., the power of the zero frequency. For the speckle contrast, the fraction of Doppler shifted light affects the contrast more pronounced for long exposure times. In the standard model, the fraction of nonDoppler shifted light increased from $28 \%$ for a vessel diameter of $5 \mu \mathrm{m}$ to $52 \%$ for a vessel diameter of $40 \mu \mathrm{m}$. The results for vessel diameters between 5 and $100 \mu \mathrm{m}$ can be seen in Fig. 4(a).

By calculating the Doppler power spectrum and the contrast from the modeled optical Doppler spectra, perfusion estimates for LDF [Eq. (6)] as well as for LSCI [Eq. (7), $n=1$ or 2, $6 \mathrm{~ms}$ exposure time] were also calculated. The relative decrease in the perfusion estimate for a vessel diameter of $40 \mu \mathrm{m}$ compared to the perfusion estimate for a vessel diameter of $5 \mu \mathrm{m}$ was $26 \%$ for LDF perfusion, whereas it was $55 \%$ and $65 \%$, respectively, for LSCI perfusion calculated with $1 / K-1$ and $1 / K^{2}-1$, respectively, for this particular example. The effect is presented in Fig. 4(b) for vessel diameters between 5 and $100 \mu \mathrm{m}$. 

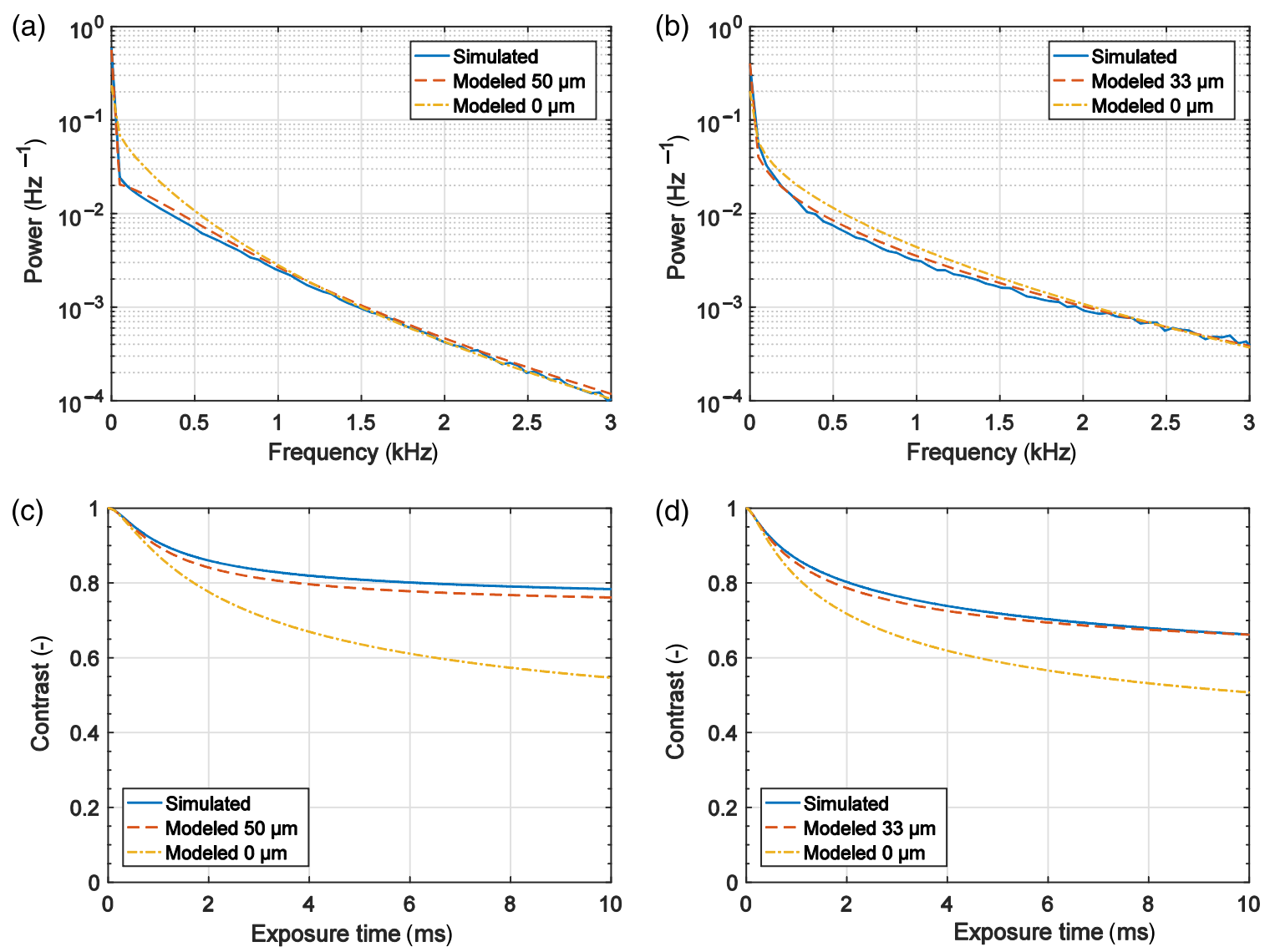

Fig. 3 ( $a$ and b) Optical Doppler spectra and (c and d) speckle contrast as a function of exposure time from the discrete vessel validation simulation (solid) and calculated from homogeneous model. Spectra are given when including the vessel packaging effect (dashed) and when not, i.e., modeling homogeneously distributed blood (dash-dotted), respectively. In (a) and (c), the simulated model contained 200 vessels with $50 \mu \mathrm{m}$ diameter. In (b) and (d), the simulated model contained 2000 models with a gamma distribution of vessel diameters, average diameter $33 \mu \mathrm{m}$.

In order to study the vessel packaging effect in more than one specific example, 2000 random models were generated with random epidermis thickness, scattering properties, fraction of melanin, blood tissue fractions, oxygen saturation, and speed distribution, i.e., parameters $t_{\text {epi }}, \mu_{\mathrm{s}}^{\prime}, f_{\text {mel }}, f_{\text {blood }, 1}, f_{\text {blood }, 2}$, $S_{\mathrm{O}_{2}}$, and $P_{v}$, see Table 1 . The LDF and LSCI perfusion estimates were calculated both when the models contained homogenously distributed blood in the layers, and when the blood was modeled to be located in vessels with $40-\mu \mathrm{m}$ average vessel diameter. The average perfusion decrease when the vessel packaging effect for $40 \mu \mathrm{m}$ average vessel diameter was included in the model was $25 \%, 43 \%$, and $52 \%$, respectively, for LDF perfusion and LSCI perfusion calculated with $1 / K-1$ and $1 / K^{2}-1$. There was a correlation between the magnitude of the vessel packaging
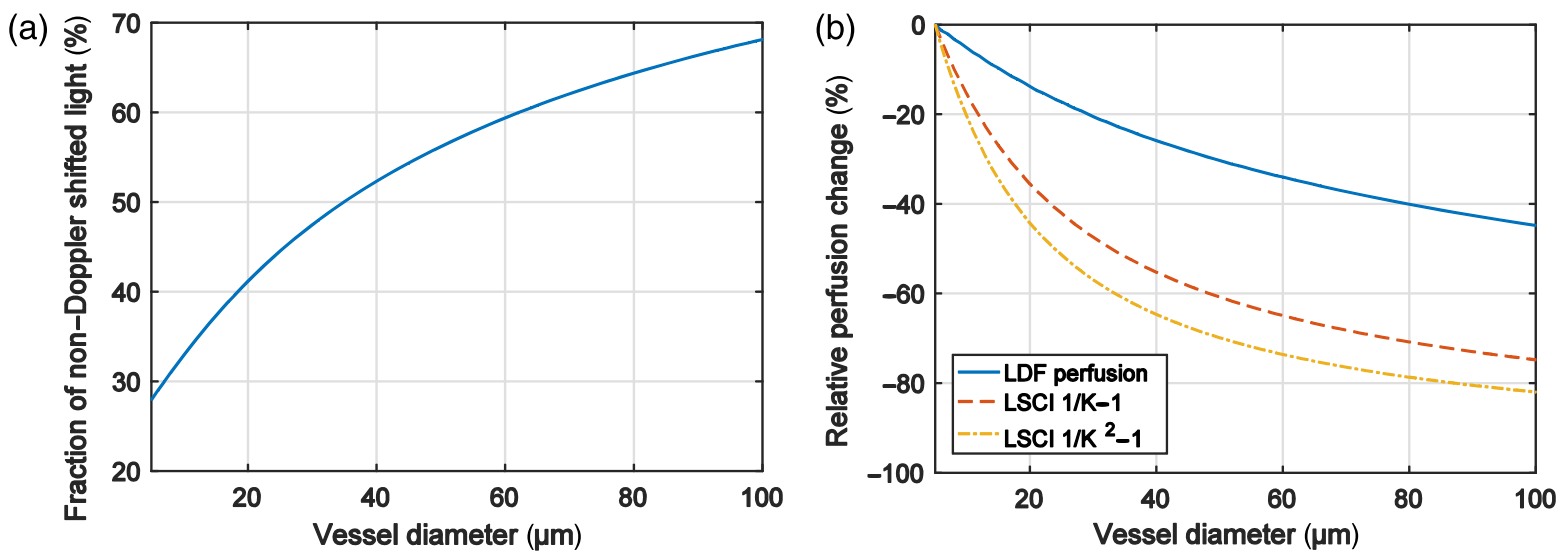

Fig. 4 (a) Fraction of non-Doppler shifted light as a function of vessel diameter. (b) Relative perfusion decrease compared to perfusion for $5 \mu \mathrm{m}$ diameter as a function of vessel diameter. 


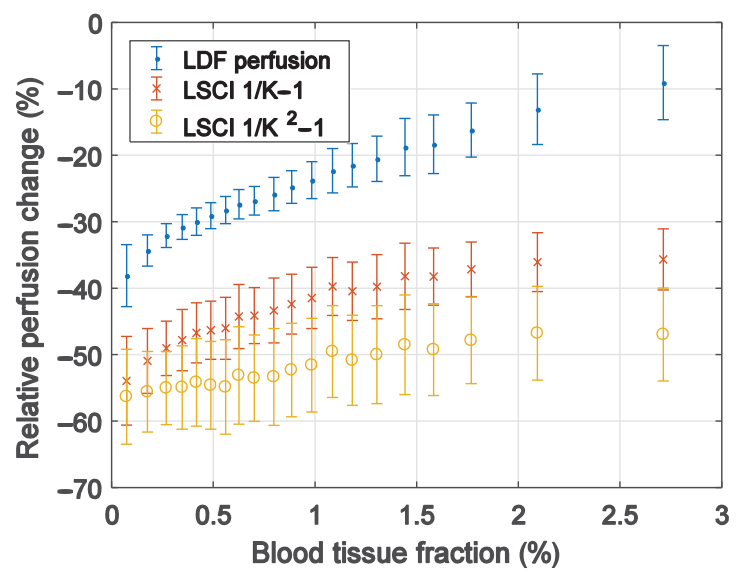

Fig. 5 Vessel packaging effect with a $40-\mu \mathrm{m}$ average vessel diameter in 2000 random models. The data were sorted according to blood tissue fraction and divided into 20 groups. The data points represent the average perfusion change as compared to homogeneously distributed blood, with the error bars showing the standard deviation.

effect and the blood tissue fraction, where the effect was strongest for low blood concentrations. These findings are summarized in Fig. 5.

\section{Discussion}

We have shown how laser speckle-based methods are affected by the fact that blood is confined in blood vessels rather than being homogenously distributed in the tissue. The magnitude of the effect is striking, not at least for LSCI where the estimated perfusion is reduced about $50 \%$ when the average vessel diameter is $40 \mu \mathrm{m}$ as compared to that for homogeneously distributed blood. The comparison with the Monte Carlo simulations containing 200 to 2000 individual blood vessels shows that we can model the effect well using the principles described in Sec. 2.2. The differences seen between simulated and modeled Doppler power spectra and contrast curves (Fig. 3) depend on some approximations in the modeling and the random heterogeneity in the simulated models containing discrete vessels.

The range of vessel diameters used in the simulated models covers those found in real skin tissue. Braverman ${ }^{26}$ reports that the cutaneous microcirculation in the upper dermis contains arterioles with a diameter of 17 to $26 \mu \mathrm{m}$, capillaries with a diameter of 4 to $6 \mu \mathrm{m}$, and venules with a diameter of 8 to $26 \mu \mathrm{m}$. In the deeper dermis, arterioles and the venules are larger having a typical diameter of $50 \mu \mathrm{m}$. Rarely, arterioles with a diameter of $100 \mu \mathrm{m}$ can be found.

There are three major causes for the vessel packaging effect in LDF and LSCI. First, less light is Doppler shifted when the light is confined in blood vessels rather than being homogeneously distributed. Second, light that is Doppler shifted in vessels is multiple shifted to a higher extent as compared to a homogenous model. Third, light that is multiple Doppler shifted within one vessel is Doppler shifted by red blood cells moving with similar speed, rather than with the speed profile of the whole microvascular model. The third effect may not be as intuitive as the first and second, but it turns out that it is important for good agreement with Monte Carlo simulations with highly different speed in different vessels, for example, in the model with 2000 individual vessels of varying diameter and flow speed. It should also be noted that the vessel packaging in diffuse reflectance spectroscopy is caused by absorption effects, which is a different cause. LDF and LSCI are generally performed at wavelengths with relatively low red blood cell light absorption, and the vessel packaging effect for these techniques is therefore not related to absorption.

It is obvious from the results that LSCI perfusion estimates are more dependent on the vessel diameter than LDF perfusion estimates. That is because LSCI perfusion is generally more affected by the amount of Doppler shifted light and less affected by the size of the Doppler shifts, as compared to LDF perfusion. ${ }^{17}$ We have previously shown that LSCI is, in general, more sensitive to variations in nonflow related tissue parameters than LDF, such as epidermis thickness, volume fraction of melanin, and scattering properties. ${ }^{17}$

This study shows that it is important to be aware of the vessel packaging effect in laser speckle-based methods, especially when comparing measurements from different sites that may contain vessels of significant different size. In the general case, it is not possible to estimate the average vessel diameter in the sampling volume when using LDF and LSCI. Hence, it will not be possible to compensate for the vessel packaging effect. An exception is when individual blood vessels are imaged and their diameter can be approximated, for example, when imaging the brain with removed skull bone or when using microscopy. In these cases, strategies for compensating for multiple Doppler shifts have been proposed by Kazmi et al. ${ }^{27}$ and Nadort et al. ${ }^{28}$

One possible way of estimating and compensating for the vessel packaging effect in LDF and LSCI is to merge these techniques with white-light spectroscopic techniques. This has been demonstrated to function in the Perimed PF 6000 EPOS system (EPOS is an acronym for enhanced perfusion and oxygen saturation), where LDF is combined with diffuse reflectance spectroscopy (DRS) using the same model, as presented in Sec. 2.2. ${ }^{12}$ The average vessel diameter is estimated using DRS spectra in that system and is thus accounted for in the estimated speed resolved perfusion.

\section{Disclosures}

No conflicts of interest, financial or otherwise, are declared by the authors.

\section{Acknowledgments}

This study was financially supported by the Swedish Research Council (Grant No. 2014-6141) and by Sweden's innovation agency VINNOVA via the program MedTech4Health (Grant No. 2016-02211).

\section{References}

1. I. Fredriksson, M. Larsson, and T. Strömberg, "Laser Doppler flowmetry," in Microcirculation Imaging, M. J. Leahy, Ed., pp. 67-86, WileyBlackwell, Weinheim (2012).

2. D. Briers et al., "Laser speckle contrast imaging: theoretical and practical limitations," J. Biomed. Opt. 18(6), 066018 (2013).

3. H. Obrig and A. Villringer, "Beyond the visible-imaging the human brain with light," J. Cereb. Blood Flow Metab. 23(1), 1-18 (2003).

4. A. A. Stratonnikov and V. B. Loschenov, "Evaluation of blood oxygen saturation in vivo from diffuse reflectance spectra," J. Biomed. Opt. 6(4), 457-467 (2001).

5. H. Karlsson et al., "Can a one-layer optical skin model including melanin and inhomogeneously distributed blood explain spatially resolved diffuse reflectance spectra?" Proc. SPIE 7896, 78962Y (2011).

6. I. Fredriksson, M. Larsson, and T. Strömberg, "Inverse Monte Carlo method in a multilayered tissue model for diffuse reflectance spectroscopy," J. Biomed. Opt. 17(4), 047004 (2012). 
7. R. Hennessy, M. K. Markey, and J. W. Tunnell, "Impact of one-layer assumption on diffuse reflectance spectroscopy of skin," J. Biomed. Opt. 20(2), 027001 (2015).

8. L. Svaasand et al., "Therapeutic response during pulsed laser treatment of port-wine stains: dependence on vessel diameter and depth in dermis," Lasers Med. Sci. 10(4), 235-243 (1995).

9. R. L. P. van Veen, W. Verkruysse, and H. J. C. M. Sterenborg, "Diffusereflectance spectroscopy from 500 to $1060 \mathrm{~nm}$ by correction for inhomogeneously distributed absorbers," Opt. Lett. 27(4), 246-248 (2002).

10. I. Fredriksson, M. Larsson, and T. Strömberg, "Accuracy of vessel diameter estimated from a vessel packaging compensation in diffuse reflectance spectroscopy," Proc. SPIE 8087, 80871M (2011).

11. G. M. Palmer and N. Ramanujam, "Monte Carlo-based inverse model for calculating tissue optical properties. Part I: theory and validation on synthetic phantoms," Appl. Opt. 45(5), 1062-1071 (2006).

12. I. Fredriksson et al., "Inverse Monte Carlo in a multilayered tissue model: merging diffuse reflectance spectroscopy and laser Doppler flowmetry," J. Biomed. Opt. 18(12), 127004 (2013).

13. M. Ewerlöf, M. Larsson, and E. G. Salerud, "Spatial and temporal skin blood volume and saturation estimation using a multispectral snapshot imaging camera," Proc. SPIE 10068, 1006814 (2017).

14. I. Fredriksson, M. Larsson, and T. Strömberg, "Absolute flow velocity components in laser Doppler flowmetry," Proc. SPIE 6094, 60940A (2006).

15. I. Fredriksson, M. Larsson, and T. Strömberg, "Optical microcirculatory skin model: assessed by Monte Carlo simulations paired with in vivo laser Doppler flowmetry," J. Biomed. Opt. 13(1), 014015 (2008).

16. I. Fredriksson, M. Larsson, and T. Strömberg, "Model-based quantitative laser Doppler flowmetry in skin," J. Biomed. Opt. 15(5), 057002 (2010).

17. I. Fredriksson and M. Larsson, "On the equivalence and differences between laser Doppler flowmetry and laser speckle contrast analysis," J. Biomed. Opt. 21(12), 126018 (2016).

18. K. Khaksari and S. J. Kirkpatrick, "Combined effects of scattering and absorption on laser speckle contrast imaging," J. Biomed. Opt. 21(7), 076002 (2016).
19. K. Khaksari and S. J. Kirkpatrick, "Laser speckle contrast imaging is sensitive to advective flux," J. Biomed. Opt. 21(7), 076001 (2016).

20. M. A. Davis et al., "Sensitivity of laser speckle contrast imaging to flow perturbations in the cortex," Biomed. Opt. Express 7(3), 759-775 (2016).

21. L. O. Reynolds and N. J. McCormick, "Approximate two-parameter phase function for light scattering," J. Opt. Soc. Am. 70(10), 1206-1212 (1980).

22. P. N. Pusey and W. van Megen, "Dynamic light scattering by nonergodic media," Phys. A 157(2), 705-741 (1989).

23. H. Furukawa and S. Hirotsu, "Dynamic light scattering from static and dynamic fluctuations in inhomogeneous media," J. Phys. Soc. Jpn. 71(12), 2873-2880 (2002).

24. K. Schatzel, "Noise on photon correlation data. I. Autocorrelation functions," Quantum Opt. 2(4), 287-305 (1990).

25. P. Zakharov et al., "Quantitative modeling of laser speckle imaging," Opt. Lett. 31(23), 3465-3467 (2006).

26. I. M. Braverman, "The cutaneous microcirculation," J. Invest. Dermatol. Symp. Proc. 5(1), 3-9 (2000).

27. S. M. Kazmi et al., "Flux or speed? Examining speckle contrast imaging of vascular flows," Biomed. Opt. Express 6(7), 2588-2608 (2015).

28. A. Nadort et al., "Quantitative blood flow velocity imaging using laser speckle flowmetry," Sci. Rep. 6, 25258 (2016).

Ingemar Fredriksson is an adjunct lecturer at the Department of Biomedical Engineering at Linköping University, Sweden, and an R\&D optics designer at Perimed $A B$, Järfälla-Stockholm, Sweden. His research focuses on modeling and model-based analysis of laser speckle based and spectroscopic techniques with applications within monitoring and imaging of microcircular blood flow and metabolic processes.

Marcus Larsson is a senior lecturer at the Department of Biomedical Engineering, Linköping University, Sweden. His research is focused on theoretical and applied biomedical optics for tissue characterization using speckle-based techniques and steady-state spectroscopic techniques. 